\title{
Correlation functions of atomic nuclei in Lattice QCD
}

\author{
Jana Günther* \\ University of Wuppertal \\ E-mail: Jana. Guenther@t-online.de
}

Bálint C. Tóth

University of Wuppertal

E-mail: tothbalinteszofi.elte.hu

Lukas Varnhorst*

University of Wuppertal

E-mail: L.Varnhorstet-online.de

\begin{abstract}
To determine the mass of atomic nuclei and other multi-baryon systems in lattice QCD one has to calculate the correlation functions of suitable combinations of quark field operators. However, the calculation of these correlation functions requires the evaluation of a large number of Wick contractions, which scales as the factorial of the number of nucleons in the system. We explore the possibilities to reduce the computational effort for the evaluation of correlation functions of atomic nuclei by exploiting certain symmetries of the systems. We discuss a recursive approach which respects these symmetries for the simplest case of identical quark sources. After that we discuss generalizations of the recursive algorithm. These generalizations include baryons from different sources and sinks and the projections to specific spin states. The construction of atomic nuclei as a special case is presented. The computational cost for the recursive construction of correlation functions of atomic nuclei is compared with the cost of other techniques.
\end{abstract}

31st International Symposium on Lattice Field Theory LATTICE 2013

July 29 - August 3, 2013

Mainz, Germany

\footnotetext{
*Speaker.
} 


\section{Introduction}

To study multi-baryon states in lattice QCD one needs to calculate correlation functions of the form

$$
\left[C^{(N)}\right]_{\alpha_{1} \alpha_{2} \ldots \alpha_{N}}^{\alpha_{1}^{\prime} \alpha_{2}^{\prime} \ldots \alpha_{1}^{\prime}}\left(\vec{x}_{1}, \vec{x}_{2}, \ldots, \vec{x}_{N}, t\right)=\left\langle\prod_{k=1}^{N} B_{\alpha_{k}}\left(\vec{x}_{k}, t\right) \prod_{l=1}^{N} \bar{B}^{\alpha_{l}^{\prime}}(\overrightarrow{0}, 0)\right\rangle .
$$

Here the $B$ and $\bar{B}$ are interpolating fields with the quantum numbers of some baryons. These operators are given by

$$
\begin{aligned}
& B_{\alpha}=\varepsilon_{a b c}\left(\Gamma_{1}\right)_{\alpha \beta}\left(q_{1}\right)_{\beta ; a}\left[\left(q_{2}\right)_{\gamma ; b}\left(\Gamma_{2}\right)_{\gamma \delta}\left(q_{3}\right)_{\delta ; c}\right] \\
& \bar{B}^{\alpha}=\varepsilon^{a b c}\left(\Gamma_{1}\right)^{\alpha \beta}\left(\bar{q}_{1}\right)^{\beta ; a}\left[\left(\bar{q}_{2}\right)^{\gamma ; b}\left(\Gamma_{2}\right)^{\gamma \delta}\left(\bar{q}_{3}\right)^{\delta ; c}\right],
\end{aligned}
$$

in terms of the quark fields. Here and throughout in the paper Latin indices correspond to color degrees of freedom (DoFs) while Greek indices correspond to the spin DoFs associated with the quark operators. For notational convenience all upper indices correspond to quark operators at the source while lower indices correspond to quark operators at the sink. The choice of $\Gamma_{1}=\mathbb{1}$ and $\Gamma_{2}=C \gamma_{5}$ yields the interpolating operators for the proton with $\left(q_{1}, q_{2}, q_{3}\right)=(u, u, d)$ and for the neutron with $\left(q_{1}, q_{2}, q_{3}\right)=(d, u, d)$. This allows the study of atomic nuclei in Lattice QCD. In principle the tools for such studies are at hand and several calculations in order to examine light nuclei $[1,2,3]$ and the nuclear force $[4,5,6]$ have been performed recently.

If one attempts to evaluate the expression in equation (1.1) via the Wick theorem in a naïve way, one is faced with $\prod_{f} n_{f}$ ! contractions. In this formula the product goes over all flavors of quarks which appear in $\bar{B}$ and $B$ and $n_{f}$ denotes the number of quarks of flavor $f$. In the case of nuclei with atomic mass number $A$ for each contraction there are $6^{A} 4^{A}$ combination of spinor- and color-indices which must be evaluated. The evaluation of all these contributions in a naïve way becomes quickly unfeasible even for moderate system sizes.

For the related, but somewhat simpler case, where the system consists of a large number of mesons, several efficient methods to calculate the correlation functions exist [7, 8]. The most recent of these techniques allows for the study of systems containing up to 72 pions [8]. For systems comprised of baryons there has also been substantial progress recently in reducing this computational challenge. In [1] the number of contractions has been reduced significantly by exploiting the permutation symmetry of the quark operators. A further improvement has been achieved in [9], where the combined permutations of color and spin indices are used to create a unified list of independent contractions. While this method reduces the amount of contractions to be evaluated on each gauge configuration significantly, the creation of the list of independent contractions remains difficult. This is due to the fact that the full set of possible contractions, which scales factorially and exponentially in the number of quarks, has to be applied once to determine the coefficients in the list. For small systems it is possible to carry out this calculation once, but it becomes quickly impractical for larger systems. The method proposed in [10] besides being able to consider multiple source locations, brings an improvement by generating the list of terms to be contracted recursively. The determinant algorithm of [10] can further reduce the computational cost in the case of certain large nuclei by transforming the factorially scaling task of calculating Wick-contractions into the polynomially scaling task of calculating determinants. 
In this work a recursive algorithm for the evaluation of the above defined correlation functions is discussed. This method, which is based on the unified contraction algorithm, was first introduced and described in more detail in [11].

The organization of this paper is as follows: In section 2 the unified contraction algorithm is described. After that in section 3 properties of antisymmetric tensors are discussed and a method for the recursive constructions of these tensors is introduced. It is followed in section 4 by a description of the recursive algorithm in the case of a single quark source. This algorithm is generalized in section 5. The spectial case of atomic nuclei is discussed in section 6 and a comparison with other methods is given. The paper is summarized in section 7 .

\section{The unified contraction algorithm}

In this work the unified contraction algorithm, which was introduced in [9], is reviewed in order to allow for a self contained presentation. Important ingredients for the unified contraction algorithms are baryon blocks. These blocks are defined as

$$
f_{B}^{q_{1}, q_{2}, q_{3}}(t, \delta ; \alpha, \beta, \gamma ; a, b, c)=\sum_{\vec{x}} s(\vec{x})\left\langle B_{\delta}(\vec{x}, t) \cdot \bar{q}_{1}^{\alpha ; a} \bar{q}_{2}^{\beta ; b} \bar{q}_{3}^{\gamma, c}\right\rangle .
$$

Thus they represent the propagation of three quarks $q_{1}, q_{2}, q_{3}$ at the source to a baryon $B$ at the sink. Such blocks have been used successfully in other works $[1,2,3,4,5,6]$. Because the blocks already contain some permutations the usage of blocks alone may already reduce the number of contractions to evaluate. Furthermore it is possible to construct baryon blocks with complicated spatial structure to increase the overlap with the desired states or to project individual baryons to specific momenta. A graphical representation of the blocks corresponding to protons and neutrons can be found in figure 1 .

It is convenient to introduce combined spinor-color indices $\xi$ and to rewrite the baryon blocks as

$$
f_{B}^{q_{1}, q_{2}, q_{3}}\left(t, \delta ; \xi^{\left(q_{1}\right)}, \xi^{\left(q_{2}\right)}, \xi^{\left(q_{3}\right)}\right)=\sum_{\vec{x}} s(\vec{x})\left\langle B_{\delta}(\vec{x}, t) \cdot \bar{q}_{1}^{\xi^{\left(q_{1}\right)}} \bar{q}_{2}^{\xi^{\left(q_{2}\right)}} \bar{q}_{3}^{\xi^{\left(q_{3}\right)}}\right\rangle .
$$

The indices $\xi$ are in the range from 1 to $4 N_{c}=12$.

In order to express the complete correlation function in terms of the baryon blocks objects $G$ can be defined, which contain the remaining Dirac- and color-structure at the source. These objects

$$
G^{B}\left(\alpha ; \xi^{\left(q_{1}\right)}, \xi^{\left(q_{2}\right)}, \xi^{\left(q_{3}\right)}\right):=\left(\Gamma_{1}\right)^{\alpha \beta\left(\xi^{\left(q_{1}\right)}\right)}\left(\Gamma_{2}\right)^{\beta\left(\xi^{\left(q_{2}\right)}\right) \beta\left(\xi^{\left(q_{3}\right)}\right)} \varepsilon^{c\left(\xi^{\left(q_{1}\right)}\right) c\left(\xi^{\left(q_{2}\right)}\right) c\left(\xi^{\left(q_{3}\right)}\right)}
$$

are also visualized in figure 1. By combining the objects $G$ and the baryon blocks $f$ an expression

$$
\begin{aligned}
& {\left[C^{(N)}\right]_{\delta_{1}, \delta_{2}, \ldots, \delta_{N}}^{\alpha_{1}, \alpha_{2}, \ldots, \alpha_{N}}(t)=} \\
& \sum_{\sigma \in \Sigma} f_{B_{1}}^{q_{1}, q_{2}, q_{3}}\left(t, \delta_{1} ; \xi_{1}^{\left(q_{1}\right)}, \xi_{2}^{\left(q_{2}\right)}, \xi_{3}^{\left(q_{3}\right)}\right) \ldots f_{B_{N}}^{q_{1}, q_{2}, q_{3}}\left(t, \delta_{N} ; \xi_{3 N-2}^{\left(q_{1}\right)}, \xi_{3 N-1}^{\left(q_{2}\right)}, \xi_{3 N}^{\left(q_{3}\right)}\right) \\
& \quad \cdot G^{B_{1}}\left(\alpha_{1} ; \xi_{\sigma(1)}^{\left(q_{1}\right)}, \xi_{\sigma(2)}^{\left(q_{2}\right)}, \xi_{\sigma(3)}^{\left(q_{3}\right)}\right) \ldots G^{B_{N}}\left(\alpha_{N} ; \xi_{\sigma(3 N-2)}^{\left(q_{1}\right)}, \xi_{\sigma(3 N-1)}^{\left(q_{2}\right)}, \xi_{\sigma(3 N)}^{\left(q_{3}\right)}\right) \operatorname{sgn}(\sigma) .
\end{aligned}
$$

for the complete correlation function can be obtained. The set $\Sigma$ containes all permutations, which permute indices which correspond to identical quark flavors. 

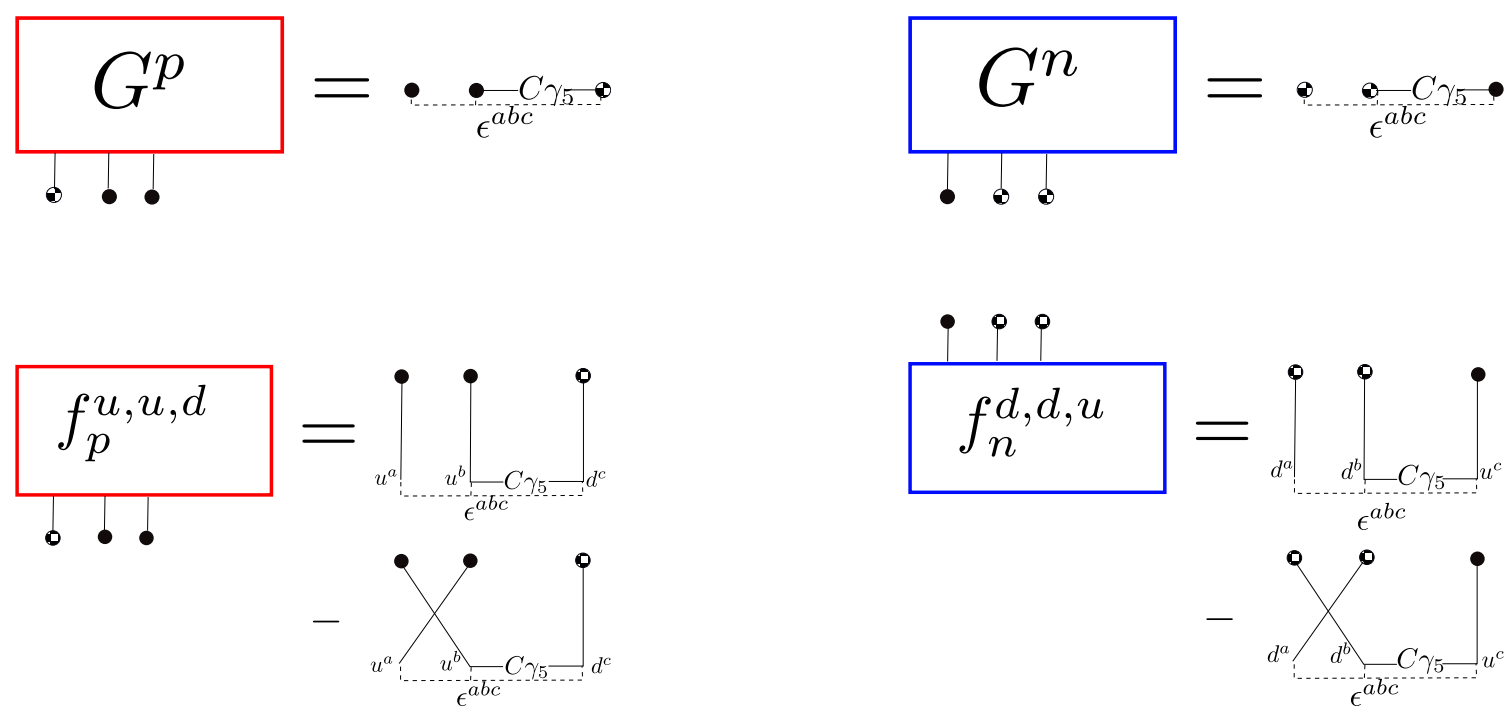

Figure 1: A graphic representation of the baryon blocks $f$ and the $G$-tensors used for the construction of correlation functions of atomic nuclei. Filled dots correspond to open indices related to $u$-quarks and halffilled dots correspond to open indices related to $d$-quarks. Solid lines correspond to connection by Dirac indices and dashed lines indicate the color structure.

Notably the structure of equation (2.4) does not depend on the the gauge configuration. It is possible to write down

$$
\begin{aligned}
& L^{(N)}\left(\alpha_{1}, \ldots, \alpha_{N} ; \xi_{1}^{\left(q_{1}\right)}, \xi_{2}^{\left(q_{2}\right)}, \xi_{3}^{\left(q_{3}\right)}, \ldots, \xi_{3 N-2}^{\left(q_{1}\right)}, \xi_{3 N-1}^{\left(q_{2}\right)}, \xi_{3 N}^{\left(q_{3}\right)}\right)= \\
& \quad \sum_{\sigma \in \Sigma} G^{B_{1}}\left(\alpha_{1} ; \xi_{\sigma(1)}^{\left(q_{1}\right)}, \xi_{\sigma(2)}^{\left(q_{2}\right)}, \xi_{\sigma(3)}^{\left(q_{3}\right)}\right) \ldots G^{B_{N}}\left(\alpha_{N} ; \xi_{\sigma(3 N-2)}^{\left(q_{1}\right)}, \xi_{\sigma(3 N-1)}^{\left(q_{2}\right)}, \xi_{\sigma(3 N)}^{\left(q_{3}\right)}\right) \operatorname{sgn}(\sigma) .
\end{aligned}
$$

which contains all $G$-tensors and all permutations and which is independent of the gauge configuration. Once the object $L$ is created it is possible to evaluate the correlation function without the need of any additional permutations via

$$
\begin{array}{r}
{\left[C^{(N)}\right]_{\delta_{1}, \delta_{2}, \ldots, \delta_{N}}^{\alpha_{1}, \alpha_{2}, \ldots, \alpha_{N}}(t)=f_{B_{1}}^{q_{1}, q_{2}, q_{3}}\left(t, \delta_{1} ; \xi_{1}^{\left(q_{1}\right)}, \xi_{2}^{\left(q_{2}\right)}, \xi_{3}^{\left(q_{3}\right)}\right) \ldots f_{B_{N}}^{q_{1}, q_{2}, q_{3}}\left(t, \delta_{N} ; \xi_{3 N-2}^{\left(q_{1}\right)}, \xi_{3 N-1}^{\left(q_{2}\right)}, \xi_{3 N}^{\left(q_{3}\right)}\right)} \\
\cdot L^{(N)}\left(\alpha_{1}, \ldots, \alpha_{N} ; \xi_{1}^{\left(q_{1}\right)}, \xi_{2}^{\left(q_{2}\right)}, \xi_{3}^{\left(q_{3}\right)}, \ldots, \xi_{3 N-2}^{\left(q_{1}\right)}, \xi_{3 N-1}^{\left(q_{2}\right)}, \xi_{3 N}^{\left(q_{3}\right)}\right) .
\end{array}
$$

It is important to note that in practice the object $L$ is sparse and therefore only a comparatively small number of terms contributes to the above expression.

\section{Treating antisymmetric tensors in a recursive way}

All independent components of a fully antisymmetric tensor $X\left(\xi_{1}, \xi_{2}, \cdots, \xi_{l}\right)$ can be uniquely described by tuples $\mathbf{A}(\boldsymbol{\xi})=(n(1), n(2), \cdots, n(k))$ where $n(i)$ is the number of occurrences of the value $i$ amongst the indices $\xi_{1}, \xi_{2}, \cdots, \xi_{l}$. Because of the antisymmetry only $n(i)=0$ and $n(i)=1$ are allowed for non-zero components. By convention the component associated with the tuple $\mathbf{A}(\xi)$ 
should be the one where the values $i$, for which $n(i)=1$, occur amongst the indices in ascending order. All other components can be constructed by rearraging the order of the indices and taking into account the sign of the permutation. For example, if $X$ is a tensor with three antisymmetric indices, each ranging from one to four, the tuple $A\{\xi\}=(1,0,1,1)$ corresponds to the component $X(1,3,4)$.

If $X$ is an antisymmetric tensor with $k$ indices and $Y$ is an antisymmetric tensor with $l$ indices, then their antisymmetric product $Z=X \bullet Y$ is a tensor with $k+l$ antisymmetric indices. The components of $Z$ are defined as

$$
(X \bullet Y)(z):=Z(z)=\sum_{z=x+y} X(x) Y(y) \operatorname{sgn}(x \mid y)
$$

where the tuples

$$
\begin{aligned}
z & =A\left\{\xi_{1}, \ldots, \xi_{k+l}\right\} \\
x & =A\left\{\xi_{1}, \ldots, \xi_{k}\right\} \\
y & =A\left\{\xi_{k+1}, \ldots, \xi_{k+l}\right\}
\end{aligned}
$$

identify the antisymmetric components and

$$
\operatorname{sgn}(x \mid y)=\prod_{\substack{i>j \\ y_{j}=1}}(-1)^{x_{i}}
$$

is the sign of the permutation that is necessary to bring the indices of the tensors $X$ and $Y$ into ascending order. If each tensor has $r$ independent groups of antisymmetric indices then each such group can be described by an individual tuple. In this case the antisymmetrized tensor is given by

$$
\begin{aligned}
&(X \bullet Y)\left(z_{1}, z_{2}, \ldots, z_{r}\right):=Z\left(z_{1}, z_{2}, \ldots, z_{r}\right) \\
&=\sum_{\substack{z_{1}=x_{1}+y_{1} \\
z_{2}=x_{2}+y_{2} \\
z_{r}=x_{r}+y_{r}}} X\left(x_{1}, x_{2}, \ldots, x_{r}\right) Y\left(y_{1}, y_{2}, \ldots, y_{r}\right) \operatorname{sgn}\left(x_{1} \mid y_{1}\right) \operatorname{sgn}\left(x_{2} \mid y_{2}\right) \ldots \operatorname{sgn}\left(x_{r} \mid y_{r}\right) .
\end{aligned}
$$

When a tensor $X^{(n)}$ can be expressed as $X^{(n)}=Y_{1} \otimes Y_{2} \otimes \cdots \otimes Y_{n}$ then the antisymmetrized form of this tensor can be obtained by $X^{(n)}=Y_{1} \bullet Y_{2} \bullet \cdots \bullet Y_{n}$ up to a normalization factor. It can be efficient to construct $X^{(n)}$ via a recursive relation of the form

$$
X^{(k)}=X^{(k-1)} \bullet Y_{k}
$$

This is especially true if one is only interested in a subset of the independent components of $X^{(n)}$. In such a case even at the intermediate stages only the construction of a subset of all possible independent components is required. An example of such a situation is depicted in figure 2. There it can be seen, that if only a single component in the last stage of the recursion is of interest at the intermediate stages only a fraction of the components need to be calculated. Therefore a drastic reduction in the number of necessary operations can be achieved. 


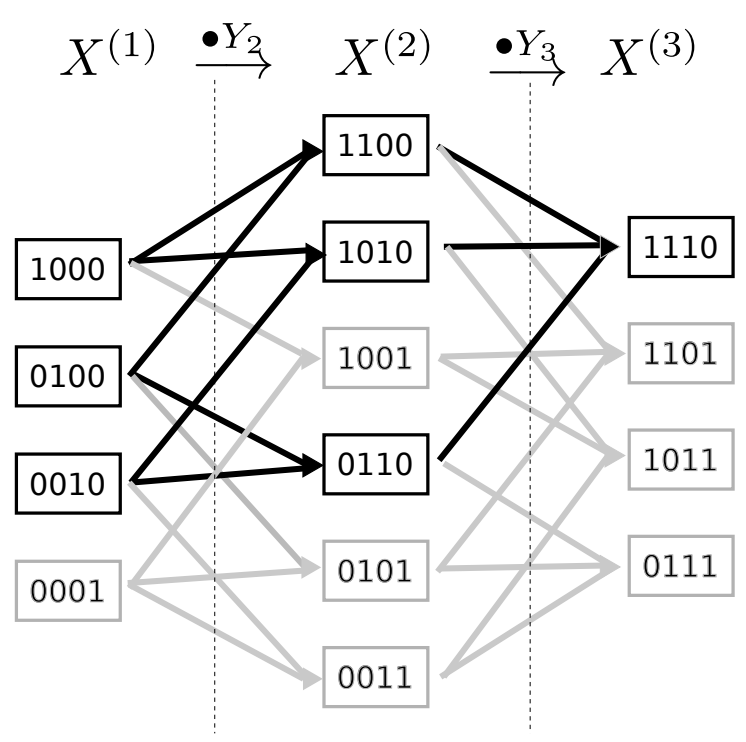

Figure 2: The recursive construction of a tensor $X^{(3)}$ with the method explained in the text. Each box represents an independent component of the given tensor and the number inside each box represents the tuple associated with this component. If only the black component of $X^{(3)}$ is needed then at the intermediate stages only the black components must be constructed and only the operations symbolized with black arrows have to be performed.

\section{Recursive construction of correlation functions using one quark source/sink}

In equation (2.6) the correlation function was reformulated in terms of an object $L$. It is antisymmetric under the excange of two indices which correspond to the same quark flavor or baryon type. Because of the antisymmetry-structure this object can be written as

$$
L\left(A^{\left(B_{a}\right)}\{\alpha\}, A^{\left(B_{b}\right)}\{\alpha\}, \ldots, A^{(u)}\{\xi\}, A^{(d)}\{\xi\}, A^{(s)}\{\xi\}\right) .
$$

Here $A^{(x)}$ corresponds to a tuple as defined in the previous section corresponding to all indices of quark flavor or baryon type $x$ and ignoring all other indices. Using this notation $L$ can be calculated via the recursion relation

$$
L^{(n+1)}=L^{(n)} \bullet G_{B_{n+1}}
$$

where $G_{B_{n+1}}$ has to be antisymmetrized in order to have the same structure as $L$.

To obtain the correlation function $L$ must be contracted with the gauge configuration dependent factors

$$
\begin{aligned}
F^{(N)}\left(\delta_{1}, \ldots \delta_{N} ; t ;\right. & \left.\xi_{1}^{\left(q_{1}\right)}, \xi_{2}^{\left(q_{2}\right)}, \xi_{3}^{\left(q_{3}\right)}, \ldots, \xi_{3 N-2}^{\left(q_{1}\right)}, \xi_{3 N-1}^{\left(q_{2}\right)}, \xi_{3 N}^{\left(q_{3}\right)}\right) \\
& :=f_{B_{1}}^{q_{1}, q_{2}, q_{3}}\left(t, \delta_{1} ; \xi_{1}^{\left(q_{1}\right)}, \xi_{2}^{\left(q_{2}\right)}, \xi_{3}^{\left(q_{3}\right)}\right) \ldots f_{B_{N}}^{q_{1}, q_{2}, q_{3}}\left(t, \delta_{N} ; \xi_{3 N-2}^{\left(q_{1}\right)}, \xi_{3 N-1}^{\left(q_{2}\right)}, \xi_{3 N}^{\left(q_{3}\right)}\right) .
\end{aligned}
$$

Because the contraction of an antisymmetric tensor with any other tensor is equal to the contraction with the antisymmetrized version of this other tensor it is useful to define an antisymmetrized version of $F$ called $F_{-}$which has the form

$$
F_{-}^{(n)}\left(A^{\left(B_{a}\right)}\{\delta\}, A^{\left(B_{b}\right)}\{\delta\}, \ldots, A^{(u)}\{\xi\}, A^{(d)}\{\xi\}, A^{(s)}\{\xi\}\right) .
$$


This antisymmetrized tensor can be calculated using the recursion

$$
F_{-}^{(n+1)}=F_{-}^{(n)} \bullet f_{B_{n+1}}^{q_{1}, q_{2}, q_{3}} .
$$

Once $F_{-}^{(n)}$ has been constructed the correlation function can be written as

$$
\begin{aligned}
& C^{(N)}\left(t ; A^{\left(B_{a}\right)}\{\delta\}, \ldots, A^{(u)}\{\alpha\}, \ldots\right) \\
& \quad=\frac{1}{\mathscr{N}} \sum_{\substack{A^{(q)}\{\xi\} \\
q \in\{u, d, s\}}} F_{-}^{(N)}\left(A^{\left(B_{a}\right)}\{\delta\}, \ldots, A^{(u)}\{\xi\}, \ldots\right) \cdot L^{(N)}\left(A^{\left(B_{a}\right)}\{\alpha\}, \ldots, A^{(u)}\{\xi\}, \ldots\right)
\end{aligned}
$$

with the normalization factor

$$
\mathscr{N}=n_{q_{a}} ! n_{q_{b}} ! \ldots\left(n_{B_{a}} ! n_{B_{b}} ! \ldots\right)^{2},
$$

where $n_{q_{i}}$ is the number of quarks of flavor $q_{i}$ and $n_{B_{i}}$ is the number of baryons of type $B_{i}$ in the system. The sum goes over all possible values of the tuples $A^{(q)}\{\xi\}$. Thus only tuples corresponding to quark indices and not to baryon indices are summed.

\section{Generalizations}

The procedure described in the previous section can only be applied to simple systems containing up to 12 quarks per flavor and might not be optimal if not all spin states are of interest. It can be extended to deal with more general cases. These extensions are discussed shortly in this section.

\subsection{Several quark sources}

To allow for $N_{s}$ quark sources the construction must be modified in a way that the contractions between the object $F_{-}$and $L$ allow for quark propagation form every baryon at the sink to every quark source. This can be achieved by replacing $f$ and $G$ with the two objects

$$
\begin{gathered}
\tilde{G}^{B}\left(\chi ; \xi^{\left(q_{1}\right)}, \xi^{\left(q_{2}\right)}, \xi^{\left(q_{3}\right)}\right)=\delta^{s(\chi), s\left(\xi^{\left(q_{1}\right)}\right)} \delta^{s(\chi), s\left(\xi^{\left(q_{2}\right)}\right)} \delta^{s(\chi), s\left(\xi^{\left(q_{3}\right)}\right)} \\
\cdot G^{B}\left(\alpha(\chi) ; \kappa\left(\xi^{\left(q_{1}\right)}\right), \kappa\left(\xi^{\left(q_{2}\right)}\right), \kappa\left(\xi^{\left(q_{3}\right)}\right)\right), \\
\tilde{f}_{B}^{q_{1}, q_{2}, q_{3}}\left(t, \psi ; \xi^{\left(q_{1}\right)}, \xi^{\left(q_{2}\right)}, \xi^{\left(q_{3}\right)}\right)=\sum_{\vec{x}} s_{s(\psi)}(\vec{x})\left\langle B_{\alpha(\psi)}(\vec{x}, t) \cdot q_{1}^{\xi^{\left(q_{1}\right)}} q_{2}^{\xi^{\left(q_{2}\right)}} q_{3}^{\xi^{\left(q_{3}\right)}}\right\rangle .
\end{gathered}
$$

The $\xi$-indices are now combined color-spinor-source indices which now range from 1 to $12 N_{s}$ and $\psi$ and $\chi$ are combined baryon-type-source indices. $s(\xi)$ is the source part of $\xi, \kappa(\xi)$ is the spinor-color part and $\alpha(\psi)$ and $\alpha(\xi)$ are the spinor-parts of $\psi$ and $\chi$. The functions $s_{i}(\vec{x})$ allow for different spatial structures of the baryons at the sink side. In this formulation it is assumed that at the source side the quarks within each baryon originate from the same source. This condition can be relaxed in a straight-forward way. The objects $F_{-}$and $G$ can be constructed as before using the recursion relations form equations (4.5) and (4.2) and the correlation function can be determined 
for mutually orthogonal sources via

$$
\begin{aligned}
& C^{(N)}\left(t ; A^{\left(B_{a}\right)}\{\psi\}, \ldots, A^{(u)}\{\chi\}, \ldots\right) \\
& \quad=\frac{1}{\mathscr{N}} \sum_{\substack{A^{\left(q_{i}\right)}\{\xi\} \\
i \in\{a, b, c\}}} F_{-}^{(N)}\left(A^{\left(B_{a}\right)}\{\psi\}, \ldots, A^{(u)}\{\xi\}, \ldots\right) \cdot L^{(N)}\left(A^{\left(B_{a}\right)}\{\chi\}, \ldots, A^{(u)}\{\xi\}, \ldots\right) .
\end{aligned}
$$

\subsection{Projection to spin states}

The correlation function itself can have a large number of spinor indices and hence can have many components. Often not single components but projections of the form

$$
C_{\mathscr{M}}(t)=\mathscr{M}_{\delta_{1}, \delta_{2}, \ldots, \delta_{N}}^{\alpha_{1}, \alpha_{2}, \ldots, \alpha_{N}}\left[C^{(N)}\right]_{\delta_{1}, \delta_{2}, \ldots, \delta_{N}}^{\alpha_{1}, \alpha_{2}, \ldots, \alpha_{N}}(t)
$$

are of interest. In this case it is possible to combine the tensors $\mathscr{M}$ and $L$ before the correlation function is calculated using

$$
\begin{aligned}
\left(n_{B_{a}} ! n_{B_{b}} ! \ldots\right)^{2} L_{\mathscr{M}}\left(A^{\left(B_{a}\right)}\{\delta\}, A^{\left(B_{b}\right)}\{\delta\}, \ldots, A^{(u)}\{\xi\}, A^{(d)}\{\xi\}, A^{(s)}\{\xi\}\right) & \\
= & \sum_{A^{\left(B_{a}\right)}\{\alpha\}, A^{\left(B_{b}\right)}\{\alpha\}, \ldots} L^{(N)}\left(A^{\left(B_{a}\right)}\{\alpha\}, A^{\left(B_{b}\right)}\{\alpha\}, \ldots, A^{(u)}\{\xi\}, A^{(d)}\{\xi\}, A^{(s)}\{\xi\}\right) \\
\times & \mathscr{M}_{-}\left(A^{\left(B_{a}\right)}\{\alpha\}, A^{\left(B_{b}\right)}\{\alpha\}, \ldots, A^{\left(B_{a}\right)}\{\delta\}, A^{\left(B_{b}\right)}\{\delta\}, \ldots\right)
\end{aligned}
$$

where $\mathscr{M}_{-}$is the antiysmmetric part of $\mathscr{M}$. This modified list can be used to calculate the projected correlation function

$$
C_{\mathscr{M}}(t)=\frac{1}{\mathscr{N}} \sum_{A} F_{-}^{(N)}\left(A^{\left(B_{a}\right)}\{\delta\}, \ldots, A^{(u)}\{\xi\}, \ldots\right) \cdot L_{\mathscr{M}}\left(A^{\left(B_{a}\right)}\{\delta\}, \ldots, A^{(u)}\{\xi\}, \ldots\right) .
$$

Now the sum goes over all tuples corresponding to either quarks or baryons.

\section{Atomic nuclei}

A special case of multi-baryon systems, which is of great interest, are atomic nuclei. For the creation and annihilation of nucleons the interpolating operators

$$
\begin{aligned}
P_{\alpha} & =\varepsilon_{a b c}\left(\Gamma_{1}\right)_{\alpha \beta} u_{\beta ; a}\left[u_{\gamma ; b}\left(\Gamma_{2}\right)_{\gamma \delta} d_{\delta ; c}\right], \\
N_{\alpha} & =\varepsilon_{a b c}\left(\Gamma_{1}\right)_{\alpha \beta} d_{\beta ; a}\left[u_{\gamma ; b}\left(\Gamma_{2}\right)_{\gamma \delta} d_{\delta ; c}\right] \\
\bar{P}^{\alpha} & =\varepsilon^{a b c}\left(\Gamma_{1}\right)^{\alpha \beta} \bar{u}^{\beta ; a}\left[\bar{u}^{\gamma ; b}\left(\Gamma_{2}\right)^{\gamma \delta} \bar{d}^{\delta ; c}\right], \\
\bar{N}^{\alpha} & =\varepsilon^{a b c}\left(\Gamma_{1}\right)^{\alpha \beta} \bar{d}^{\beta ; a}\left[\bar{u}^{\gamma ; b}\left(\Gamma_{2}\right)^{\gamma \delta} \bar{d}^{\delta ; c}\right]
\end{aligned}
$$

are commonly used. If $n_{P}$ and $n_{N}$ denote the number of protons and the number of neutrons in a system the recursion relations for $F_{-}$and $L$ can be brought to the form

$$
\begin{aligned}
& L^{\left(n_{P}+1, n_{N}\right)}=L^{\left(n_{P}, n_{N}\right)} \bullet G_{P}, \\
& L^{\left(n_{P}, n_{N}+1\right)}=L^{\left(n_{P}, n_{N}\right)} \bullet G_{N}, \\
& F_{-}^{\left(n_{P}+1, n_{N}\right)}=F_{-}^{\left(n_{P}, n_{N}\right)} \bullet f_{P}^{u, u, d}, \\
& F_{-}^{\left(n_{P}, n_{N}+1\right)}=F_{-}^{\left(n_{P}, n_{N}\right)} \bullet f_{N}^{d, u, d},
\end{aligned}
$$




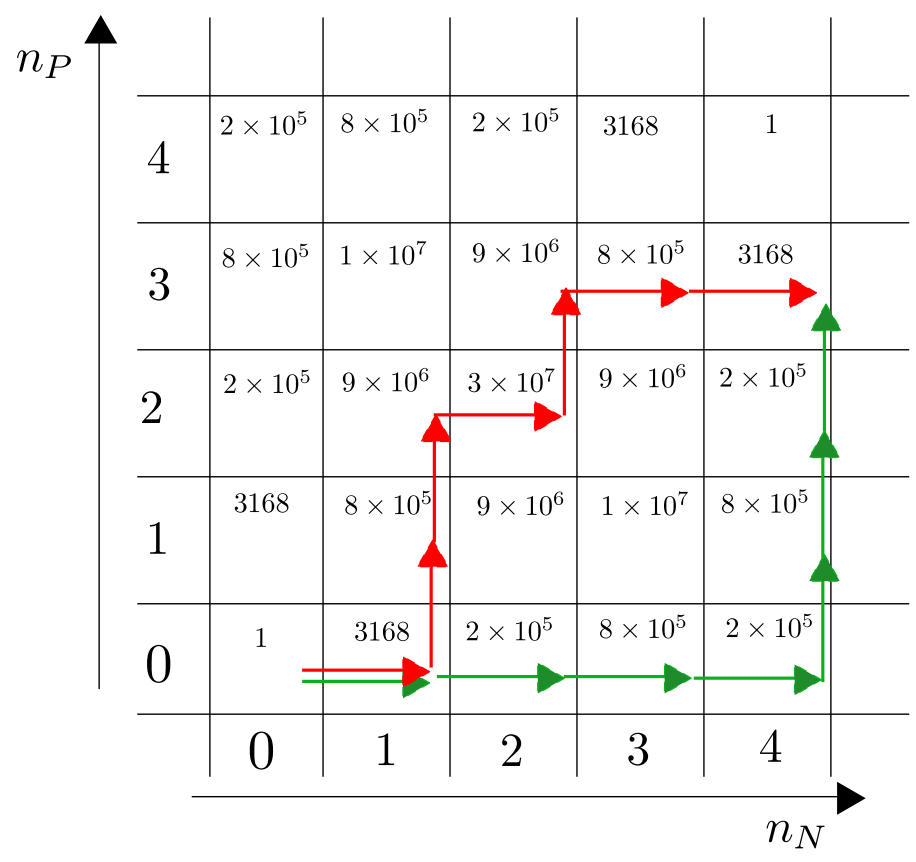

Figure 3: A scematic representation of the objects $F_{-}$used in the recursion relations for atomic nuclei. Each number corresponds to the upper bound of independent components of $F_{-}$for the indicated numbers $\left(n_{P}, n_{N}\right)$ in the reltivistic case with one quark source. The red and green arrows indicate different paths which can be used to construct the same tensor. The green path is more efficient than the red one.

with the starting conditions either

$$
L^{(0,1)}=G_{N} \quad \text { and } \quad F_{-}^{(0,1)}=f_{N}^{d, u, d}
$$

or

$$
L^{(1,0)}=G_{P} \quad \text { and } \quad F_{-}^{(1,0)}=f_{P}^{u, u, d} .
$$

For the number of independent components at each stage of the recursion the upper bound

$$
\begin{aligned}
P\left(n_{P}, n_{N}\right)=C\left(n_{P}, D-n_{P}\right) C\left(n_{N}, D-n_{N}\right) \\
\quad C\left(2 n_{P}+n_{N}, 3 D-2 n_{P}-n_{N}\right) C\left(n_{P}+2 n_{N}, 3 D-n_{P}-2 n_{N}\right),
\end{aligned}
$$

can be derived. Here $D$ is the number of effective spinor components, which is 2 for the nonrelativistic case and 4 for the relativistic case and $C\left(n_{1}, n_{2}, \cdots\right)=\left(n_{1}+n_{2}+\cdots\right) ! /\left(n_{1} ! n_{2} ! \cdots\right)$ are multinomial coefficients. In an actual computation the number of required components might be significantly smaller due to the sparse nature of $L$.

The objects $F_{-}^{\left(n_{P}, n_{N}\right)}$ (and $L^{\left(n_{P}, n_{N}\right)}$ ) can be arranged on a grid, as it is shown in figure 3. There are several ways the relations (6.2a)-(6.2d) can be applied to arrive at the same tensor. These ways however differ in their computational complexity. This is true not only when the upper bound is considered but also when the actual number of required components are taken into account. Here and in the following discussion only the computational effort for the construction of the objects $F_{-}^{\left(n_{P}, n_{N}\right)}$ are considered which dominates the calculation. All other tasks such as e.g. the contraction of $F_{-}^{\left(n_{P}, n_{N}\right)}$ with $L^{\left(n_{P}, n_{N}\right)}$ can be neglected. 


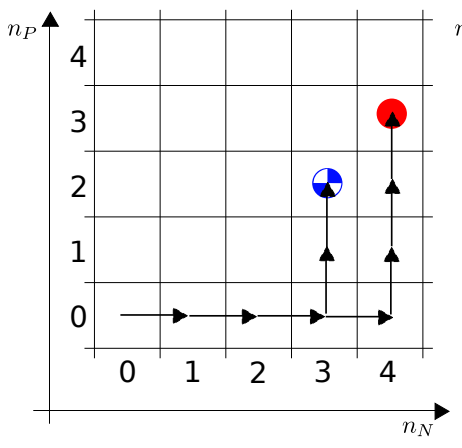

(a) $1.05 \cdot 10^{9}$ operations

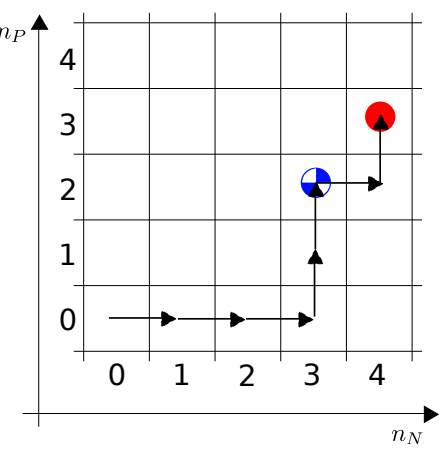

(b) $4.03 \cdot 10^{9}$ operations

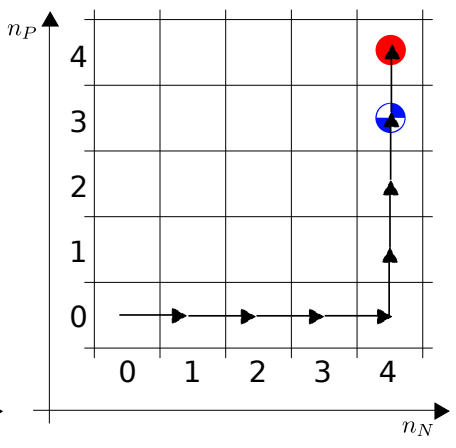

(c) $4.48 \cdot 10^{9}$ operations

Figure 4: Three representative cases for the combined calculation of two atomic nuclei. The red and blue dots represent the nuclei that are to be calculated and the arrows indicate the order in which protons and neutrons are added. In the case (a) a speedup of about $10 \%$ can be reached. In case (b) more operations are required than for the separate calculation of the indicated nuclei. In case (c) the blue nucleus can be calculated without additional effort. Therefore in this case a speedup of about $47 \%$ can be observed.

An example for two different paths is shown in figure 3. In the case of the green path all required neutrons are added first and the protons are added afterwards. In the case of the red path additions of the two different types of nucleons are mixed. It was found that it is always advantageous to add one species of nucleons first, so that the area where the number of independent components can be large is avoided. This region is particularly expensive because the reduction of the number of independent components due to the antisymmetry structure is least effective in this region.

Given that the objects $F_{-}^{\left(n_{P}, n_{N}\right)}$ and $L^{\left(n_{P}, n_{N}\right)}$ are constructed recursively it is natural to ask, whether it is advantageous to combine the calculation of several nuclei. The answer to this question depends on what nuclei and which spin-states are of interest. In figure 4 three possible situations are shown. It is assumed that all spin-states are of interest. In $4 \mathrm{a}$ and $4 \mathrm{~b}$ two systems with different numbers $\left(n_{P}, n_{N}\right)$ are to be calculated, namely $(2,3)$ and $(3,4)$. In the case shown in figure 4a the first three stages are common for both nuclei. In this setting a speedup of $10 \%$ compared to the separate calculation can be achieved. An alternative to this setting is shown in figure 4b. Here a path on which both nuclei are located is used. It turns out that this path results in more operations than the separate calculation of the two nuclei. In figure $4 \mathrm{c}$ the nuclei with $(4,4)$ and $(3,4)$ are to be calcluated. In this case a speedup of $47 \%$ compared to the separate calculation can be observed.

The computational cost for the construction of the tensors $F_{-}^{\left(n_{P}, n_{N}\right)}$ for all nuclei accessible with one quark source and relativistic operators is given in table 1. It can be seen, that the described approach is much more efficient than the naïve calculation for all nuclei. The gain increases with growing system size. In table 2 the recursive method is compared to the unified contraction algorithm as it is introduced in [9] for selected nuclei and spin-states. The recursive method is advantageous for all but the smallest systems.

The method of [10] uses determinants for the calculation of the quark level permutations in the correlation function for a fixed structure of color-spinor indices and spatial location of the 


\begin{tabular}{lllll}
\hline \hline$N_{P}$ & $N_{N}$ & No. of op. & Naïve No. of op. & $\eta$ \\
\hline 0 & 2 & 199584 & 15925248 & 79.8 \\
0 & 3 & 5825088 & $8.3 \times 10^{11}$ & $1.4 \times 10^{5}$ \\
0 & 4 & 54768672 & $1.1 \times 10^{17}$ & $1.9 \times 10^{9}$ \\
1 & 1 & 474048 & 11943936 & 25.2 \\
1 & 2 & 19241280 & $5.5 \times 10^{11}$ & $2.9 \times 10^{4}$ \\
1 & 3 & 109789200 & $6.7 \times 10^{16}$ & $6.1 \times 10^{8}$ \\
1 & 4 & 179769600 & $1.7 \times 10^{22}$ & $9.2 \times 10^{13}$ \\
2 & 2 & 531321120 & $5.7 \times 10^{16}$ & $1.1 \times 10^{8}$ \\
2 & 3 & 756897264 & $1.3 \times 10^{22}$ & $1.7 \times 10^{13}$ \\
2 & 4 & 291957888 & $5.3 \times 10^{27}$ & $1.8 \times 10^{19}$ \\
3 & 3 & 2905079520 & $4.9 \times 10^{27}$ & $1.7 \times 10^{18}$ \\
3 & 4 & 404946240 & $3.0 \times 10^{33}$ & $7.5 \times 10^{24}$ \\
4 & 4 & 448496928 & $2.8 \times 10^{39}$ & $6.2 \times 10^{30}$ \\
\hline \hline
\end{tabular}

Table 1: Number of operations (each operation is a complex multiplication and addition) required to compute all independent spinor components of the correlation function with $N_{P}$ protons and $N_{N}$ neutrons with one quark source and relativistic operators. Both the naïve number and the number using the recursive approach are given. $\eta$ is the gain factor, that is, the ratio of the naïve and the recursive numbers of operations.

operators at the source and at the sink. This algorithm scales as

$$
n_{u}^{3} n_{d}^{3} n_{s}^{3} \cdot N_{w} N_{w}^{\prime}
$$

where $N_{w}$ and $N_{w}^{\prime}$ are the number of such independent structures up to permutations of quarks at the source and at the sink, respectively. This algorithm is especially efficient in the case where the numbers of quarks $n_{u}, n_{d}$ and $n_{s}$ are chosen such that all possible spinor- and color-DoFs are fully saturated both at the source and at the sink. In this case $N_{w}=N_{w}^{\prime}=1$. Such combinations of operators can be found for the nuclei ${ }^{4} \mathrm{He},{ }^{8} \mathrm{Be},{ }^{12} \mathrm{C},{ }^{16} \mathrm{O}$ and ${ }^{28} \mathrm{Si}$ for which concrete results are presented in [10]. However, in the general case the numbers $N_{w}$ and $N_{w}^{\prime}$ may become very large and in fact scale exponentially as already noted in [10].

If the individual baryons are to have a complex spatial structure, which is required e.g. for the projection to a definite momentum and angular momentum, it is very difficult to find a combination of operators for which $N_{w}$ and $N_{w}^{\prime}$ remain small. In such cases the algorithm presented in this paper, which can perform the construction of baryon blocks with complex spatial structure in advance of the calculation, can be more advantageous.

\section{Summary}

A method for the construction of correlation function of multi baryon systems was described for the simplest case. This algorithm allows to construct different parts for the correlation function in a recursive way. One part is a list of contributing terms, similar to the one used in the unified contraction algorithm, which is independent of the gauge configuration. The other part is an object 


\begin{tabular}{|c|c|c|c|c|}
\hline$N_{P}$ & $N_{N}$ & spinstate & $N_{\Lambda}$ & $\eta$ \\
\hline 0 & 2 & $(0,1)$ & 5544 & 1 \\
\hline 0 & 3 & $(0,1,2)$ & 1360098 & 1.3 \\
\hline 0 & 3 & $(0,1,3)$ & 1360098 & 1.3 \\
\hline 0 & 4 & $(0,1,2,3)$ & 54768672 & 8.1 \\
\hline 1 & 1 & $(0,0)$ & 2079 & 2 \\
\hline 1 & 1 & $(1,0)$ & 2358 & 2 \\
\hline 1 & 1 & $(0,1)$ & 2358 & 2 \\
\hline 1 & 1 & $(1,1)$ & 2079 & 2 \\
\hline 1 & 2 & $(0,0,1)$ & 381978 & 3.7 \\
\hline 1 & 2 & $(1,0,1)$ & 381978 & 3.7 \\
\hline 1 & 3 & $(0,0,1,2)$ & 11717937 & 28.8 \\
\hline 1 & 3 & $(1,0,1,2)$ & 11717937 & 28.8 \\
\hline 1 & 3 & $(0,0,1,3)$ & 11717937 & 28.8 \\
\hline 1 & 3 & $(1,0,1,3)$ & 11717937 & 28.8 \\
\hline 1 & 4 & $(0,0,1,2,3)$ & 141103602 & 321.1 \\
\hline 1 & 4 & $(1,0,1,2,3)$ & 141103602 & 321.1 \\
\hline 2 & 2 & $(0,1,0,1)$ & 8541864 & 41.2 \\
\hline 2 & 3 & $(0,1,0,1,2)$ & 44343561 & 938.7 \\
\hline 2 & 3 & $(0,1,0,1,3)$ & 44343561 & 938.7 \\
\hline 2 & 4 & $(0,1,0,1,2,3)$ & 214572144 & 14767 \\
\hline 3 & 3 & $(0,1,2,0,1,2)$ & 163007703 & 17494.5 \\
\hline 3 & 3 & $(0,1,3,0,1,2)$ & 181280493 & 17842.2 \\
\hline 3 & 3 & $(0,1,2,0,1,3)$ & 181280493 & 17842.2 \\
\hline 3 & 3 & $(0,1,3,0,1,3)$ & 163007703 & 17494.5 \\
\hline 3 & 4 & $(0,1,2,0,1,2,3)$ & 293717796 & 566364 \\
\hline 3 & 4 & $(0,1,3,0,1,2,3)$ & 293717796 & 566364 \\
\hline 4 & 4 & $(0,1,2,3,0,1,2,3)$ & 448496928 & $1.6 \times 10^{7}$ \\
\hline
\end{tabular}

Table 2: The efficiency of the presented algorithm for the calculation of individual spin components with relativistic operators. $N_{\Lambda}$ is the number of operations (complex multiplications and additions) required for the construction of $F_{-} . \eta$ is approximately the ratio of the number of operations required for the unified contraction algorithm and for the algorithm presented in this paper if additions are taken as much faster than multiplications. The entries in the the tuple denoted as "spinstate" are the values of the dirac components of the correlation function.

constructed from blocks of quark propagators which has to be calculated on each gauge configuration. The algorithm exploits the antisymmetric structure of these parts at all intermediate stages. Generalizations of the algorithm like additional quark sources and projection to spin states where discussed. The important special case of atomic nuclei was described. Different orders of recursive operations where considered and the possibility to combine the calculation of several correlation functions was introduced. A comparison with alternative methods was given. 


\section{Acknowledgement}

We would like to thank Zoltán Fodor for the stimulating suggestions and continuous support throughout the project. This work is supported in part by the DFG grant SFB/TR55 and the GSI grant WF1012.

\section{References}

[1] T. Yamazaki, Y. Kuramashi, A. Ukawa (PACS-CS Collaboration), Helium Nuclei in Quenched Lattice QCD, Phys. Rev. D 81 (2010) 111504 [arXiv:0912.1383 [hep-lat]].

[2] S. R. Beane, E. Chang, S. D. Cohen, W. Detmold, H. W. Lin, T. C. Luu, K. Orginos and A. Parreno et al., Light Nuclei and Hypernuclei from Quantum Chromodynamics in the Limit of SU(3) Flavor Symmetry, Phys. Rev. D 87 (2013) 034506 [arXiv:1206.5219 [hep-lat]].

[3] T. Yamazaki, K. -i. Ishikawa, Y. Kuramashi and A. Ukawa, Helium nuclei, deuteron and dineutron in 2+1 flavor lattice QCD, Phys. Rev. D 86 (2012) 074514 [arXiv:1207.4277 [hep-lat] ].

[4] N. Ishii, S. Aoki and T. Hatsuda, The Nuclear Force from Lattice QCD, Phys. Rev. Lett. 99 (2007) 022001 [nucl-th/0611096].

[5] S. Aoki, T. Hatsuda and N. Ishii, Theoretical Foundation of the Nuclear Force in QCD and its applications to Central and Tensor Forces in Quenched Lattice QCD Simulations, Prog. Theor. Phys. 123 (2010) 89 [arXiv:0909.5585 [hep-lat]].

[6] T. Doi, S. Aoki, T. Hatsuda, Y. Ikeda, T. Inoue, N. Ishii, K. Murano, H. Nemura, K. Sasaki (HAL QCD Collaboration), Exploring Three-Nucleon Forces in Lattice QCD, Prog. Theor. Phys. 127 (2012) 723 [arXiv:1106.2276 [hep-lat]].

[7] W. Detmold and M. J. Savage, A method to study complex systems of mesons in Lattice QCD, Phys. Rev. D 82 (2010) 014511 [arXiv:1001.2768 [hep-lat]].

[8] W. Detmold, K. Orginos and Z. Shi, Lattice QCD at non-zero isospin chemical potential, Phys. Rev. D 86 (2012) 054507 [arXiv: 1205.4224 [hep-lat] ].

[9] T. Doi and M. G. Endres, Unified contraction algorithm for multi-baryon correlators on the lattice, Comput. Phys. Commun. 184 (2013) 117 [arXiv: 1205.0585 [hep-lat]].

[10] W. Detmold and K. Orginos, Nuclear correlation functions in lattice QCD Phys. Rev. D 87 (2013) 114512 [arXiv:1207.1452 [hep-lat]].

[11] J. Günther, B. C. Toth, L. Varnhorst, A recursive approach to determine correlation functions in multi-baryon systems, Phys. Rev. D 87 (2013) 094513 [arXiv:1301.4895 [hep-lat]]. 